\title{
Application of microbiological rapid on-site evaluation in respiratory intensive care units: a retrospective study
}

\author{
Yi Tao ${ }^{1,2 \#}$, Licheng Song ${ }^{1 \#}$, Han Fu ${ }^{1}$, Wei Zhang ${ }^{1}$, Yuwei Song ${ }^{1}$, Hongxiang Liu $^{1}$, Lixin Xie ${ }^{1}$, Kaifei Wang ${ }^{1}$ \\ ${ }^{1}$ College of Pulmonary and Critical Care Medicine, Chinese PLA General Hospital, Beijing, China; ${ }^{2}$ Medical School of Chinese PLA, Beijing, China \\ Contributions: (I) Conception and design: L Xie, K Wang, Yi Tao; (II) Administrative support: L Xie; (III) Provision of study materials or patients: \\ H Fu, W Zhang, Y Song, H Liu; (IV) Collection and assembly of data: Y Tao, L Song; (V) Data analysis and interpretation: Y Tao, L Song; (VI) \\ Manuscript writing: All authors; (VII) Final approval of manuscript: All authors. \\ "These authors contributed equally to this work. \\ Correspondence to: Lixin Xie, Kaifei Wang. College of Pulmonary and Critical Care Medicine, Chinese PLA General Hospital, Beijing 100091, China. \\ Email: xielx301@126.com; wangkaifei@126.com.
}

Background: To analyze the clinical value of microbiological rapid on-site evaluation (M-ROSE) in the respiratory intensive care unit (RICU) and its impact on the prognosis of critically ill patients.

Methods: We retrospectively included patients who underwent bedside bronchoalveolar lavage in the RICU of Chinese People's Liberation Army (PLA) General Hospital between January 2017 and December 2020. The patients were divided into M-ROSE and control groups according to whether bedside M-ROSE was performed with to guide the treatment, and for control group, routine treatments were administrated based on all the clinical information. The basic information, treatment methods, test indicators, and prognostic evaluation of the patients were collected and analyzed.

Results: A total of 242 patients were enrolled, including 130 patients in the M-ROSE group and 112 patients in the control group. The inflammatory indicators of the patients in the M-ROSE group decreased significantly faster after admission than those of patients in the control group, and the patients in the M-ROSE group used significantly more types of antibiotics [5 $(3.0,5.0)]$ than those in the control group [3 $(2.0,4.0)](\mathrm{P}<0.05)$. Among the patients who were on invasive mechanical ventilation, the mortality rate of the M-ROSE group was significantly lower than that of the control group $(\mathrm{P}<0.05)$. The coincidence rate of the M-ROSE results with metagenomic next-generation sequencing (mNGS) results was 66.2\%, which was higher than the coincidence rate of other laboratory results. In addition, the M-ROSE reports were available significantly faster than the smear, culture, and mNGS results.

Conclusions: M-ROSE has high diagnostic value for lower respiratory tract pathogens. The application of M-ROSE in the RICU can help to promote a decrease in patients' inflammation levels and reduce the mortality of patients on invasive mechanical ventilation.

Keywords: Microbiological rapid on-site evaluation (M-ROSE); intensive care unit; bronchoalveolar lavage; invasive mechanical ventilation; lower respiratory tract infection

Submitted Sep 08, 2021. Accepted for publication Nov 09, 2021.

doi: $10.21037 / \mathrm{atm}-21-5465$

View this article at: https://dx.doi.org/10.21037/atm-21-5465

\footnotetext{
^ ORCID: 0000-0002-7244-0086.
} 


\section{Introduction}

Pneumonia and lower respiratory tract infections were the deadliest infectious diseases and the fourth leading cause of death worldwide in 2019. For lower respiratory tract infectious diseases, the most important action is to identify the pathogens in the early stage. Bronchoalveolar lavage fluid (BALF) is a valuable specimen that provides an indication of the physiological and pathological conditions of the lower respiratory tract, distal airway, and alveoli, and suggests the pathogens of infection. Rapid and accurate cytological and pathogenic analysis of BALF can help to reveal the pathogens responsible for lower respiratory tract infections, leading to accurate use of sensitive antibacterial drugs at an early stage and improved patient prognosis (1). Rapid on-site evaluation (ROSE), including cytological ROSE (C-ROSE) and microbiological ROSE (M-ROSE), is a diagnostic interventional pulmonology technique (2). C-ROSE often accompanies the sampling process for on-site cytological interpretation and is extensively used for the diagnosis of neoplastic lesions of the respiratory system. M-ROSE is a microbial pathogenic examination technique of lung brushing and BALF specimens. The standard procedure of M-ROSE includes cytology and microorganism analysis of BALF, it is primarily used in clinical practice for rapid bedside etiological diagnosis of lower respiratory tract infections in the respiratory intensive care unit (RICU). Compared with other applications of new equipment for microbiological rapid evaluation in pneumonia, M-ROSE detection focus on the cytology, bacteria and fungi content in lower respiratory tract specimens, increased proportion of neutrophil and the appearance of neutrophil phagocytosis is strongly indicative of bacterial infections (3-5). The clinical value of M-ROSE for the diagnosis of pathogens responsible for lower respiratory tract infections has rarely been reported $(6,7)$. Therefore, this study retrospectively analyzed the clinical value of M-ROSE in the RICU and its impact on the prognosis of critically ill patients. We present the following article in accordance with the STROBE reporting checklist (available at https:// dx.doi.org/10.21037/atm-21-5465).

\section{Methods}

\section{Subjects}

A total of 242 patients who were hospitalized and underwent bedside bronchoalveolar lavage in the RICU of
Chinese PLA General Hospital between January 2017 and December 2020 were retrospectively enrolled in the study. The patients were divided into an M-ROSE group (130 cases) and a control group (112 cases) based on whether bedside M-ROSE was performed. The inclusion criteria were as follows: (I) patients aged $\geq 18$ years; (II) patients who underwent bedside bronchoalveolar lavage. The exclusion criteria were as follows: (I) hospital stay $\geq 60$ days; (II) incomplete clinical data. For patients in M-ROSE group, the BALF were analyzed by M-ROSE, and treatments were administrated based on combination of all the clinical information and M-ROSE results. For control group, routine treatments were administrated based on all the clinical information including symptoms, medical tests, histories, vital signs and so on. The number of cases in the RICU during the study period leaded to the sample size. All procedures performed in this study involving human participants were in accordance with the Declaration of Helsinki (as revised in 2013). This study was approved by the Ethics Committee of Chinese PLA General Hospital (No. 2019-083-01). Individual consent for this retrospective analysis was waived.

\section{Research methods}

Data collection: the basic information of patients was collected, including sex, age, smoking history, and basic diseases. The Acute Physiology and Chronic Health Evaluation (APACHE-II) and Sequential Organ Failure Assessment (SOFA) scores at admission were collected. Information was collected on antibiotic use during hospitalization (type and duration), specifically, the choices of antibiotics were made based on all the clinical information or combination of all the clinical information and M-ROSE results.

The time at which inflammatory indicators, including interleukin 6 (IL-6), C-reactive protein (CRP), and procalcitonin (PCT), began to decrease and the time at which these indicators returned to normal after admission were recorded. The time at which body temperature started to decrease and the time at which it returned to normal, the length of hospital stay, and the 28-day outcome were also recorded.

M-ROSE procedure: BALF was collected according to the standard procedure recommended in the guidelines (1). At least $2 \mathrm{~mL}$ of BALF was centrifuged at $300 \mathrm{~g}$ for 10 minutes. The supernatant was removed, resuspended in $0.5 \mathrm{~mL}$ of saline, and treated with Diff-Quik stain and Gram 


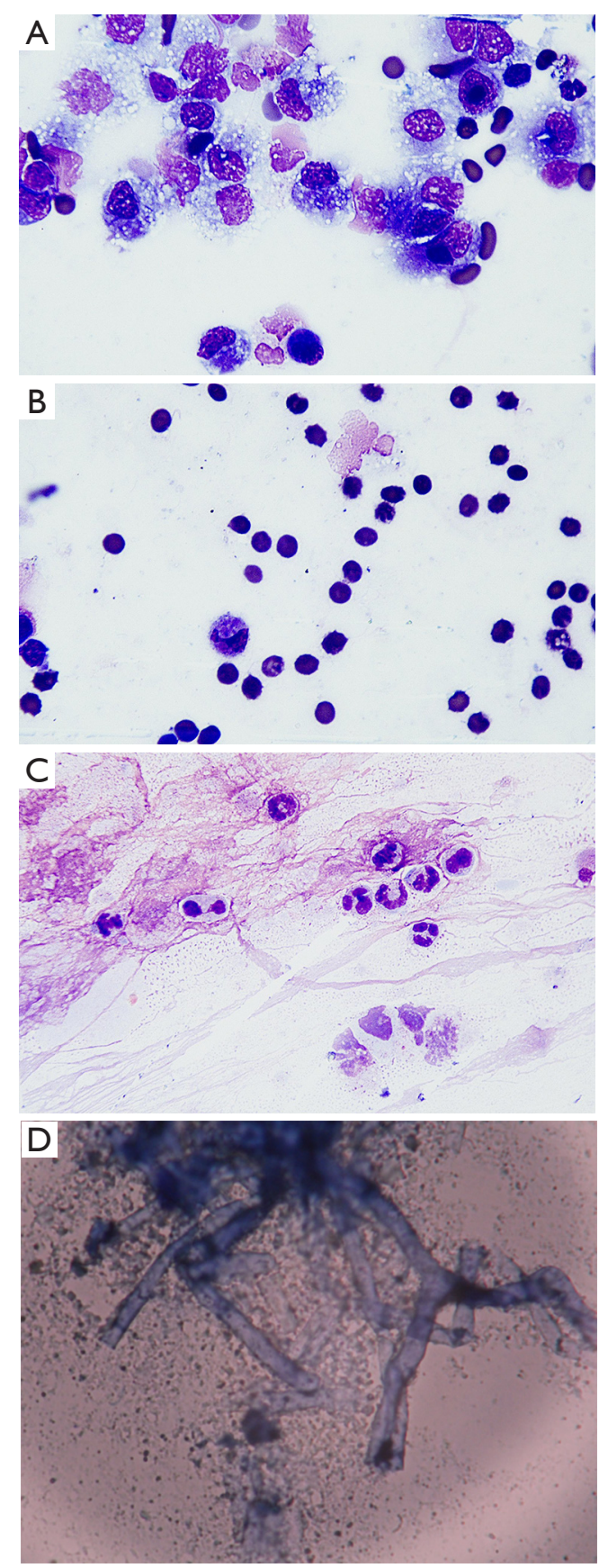

Figure 1 Microscopic BALF cell patterns and pathogens (DiffQuik stain, $\times 1,000)$. (A) Normal cell pattern; (B) hemorrhagic cell pattern; (C) infectious cell pattern; (D) invasive aspergillus. BALF, bronchoalveolar lavage fluid.

stain simultaneously according to standard procedures $(8,9)$. After drying, the slides were observed under an optical microscope. For normal BALF, the squamous epithelial cell or ciliated columnar epithelial cell proportion is less than $5 \%$. A neutrophil proportion greater than or equal to $50 \%$ strongly suggests bacterial infections, such as aspiration pneumonia or suppurative infection (1). The appearance of bacterial or fungal hyphae may be indicative of specific infections. As a result, M-ROSE results aid to determine whether the specimen was from lower respiratory tract, whether the cytology shows an infectious pattern and whether bacterial or fugus infections exist. A typical BALF microscopic image is shown in Figure 1.

The coincidence rate of the results: the ROSE results showed gram-positive cocci, gram-positive bacilli, gramnegative cocci, gram-negative bacilli, Candida, and fungus. They were compared with the laboratory analysis of the smear, culture, and metagenomic next-generation sequencing (mNGS) results from the same specimen.

Patients on invasive mechanical ventilation underwent tracheal intubation or tracheotomy, and patients on not invasive mechanical ventilation underwent nasal oxygen inhalation, high-flow nasal cannula oxygen therapy, and noninvasive mechanical ventilation.

\section{Statistical analysis}

SPSS 26.0 software was used for statistical analysis. Normality testing was performed, and measurement data that conformed to the normal distribution were expressed as the mean $\pm \mathrm{SD}$, while measurement data that did not conform to the normal distribution were expressed as $M$ (IQR). An intergroup differential analysis of measurement data was performed using the $t$-test or rank sum test, and the $\chi^{2}$ test was used for count data. The M-ROSE reporting time was compared with the smear, culture, and mNGS reporting times using a paired $t$-test, and $\mathrm{P}<0.05$ was considered a statistically significant difference.

\section{Results}

\section{Basic patient information}

There were 130 patients in the M-ROSE group, including 90 males and 40 females, with a median age of 66.5 (52.75, $80.00)$ years. There were 112 patients in the control group, including 77 males and 35 females, with a median age of 74 $(57.25,82.75)$ years. There were no significant differences in sex, age, basic diseases, APACHE-II score, or SOFA score between the M-ROSE and the control groups $(\mathrm{P}>0.05$, Table 1). 


\section{The effect of M-ROSE on the changes in inflammatory indicators and patient prognosis}

For patients enrolled in two groups, the time to the initial decrease in the inflammatory indexes IL-6 $[1(1.0,2.0)$ days], CRP [1 $(1.0,1.0)$ days], and PCT [1 $(1.0,1.0)$ days], and the time at which the PCT returned to normal [1 $(1.0,3.0)$ days] were significantly lower in the M-ROSE group than in the control group $(\mathrm{P}<0.05)$. Additionally, the M-ROSE group used significantly more types of antibiotics [5 (3.0, 5.0)] than the control group $[3(2.0,4.0)](\mathrm{P}<0.05$, Table 2), broader coverage of antibiotics based on M-ROSE results may lead to the faster decrease of inflammatory indicators of the patients in the M-ROSE group. However, there was no significant difference in the 28-day mortality

Table 1 Baseline patient characteristics

\begin{tabular}{|c|c|c|c|}
\hline Variable & M-ROSE $(n=130)$ & Control $(n=112)$ & $P$ value \\
\hline Age (years), M (IQR) & $66.5(52.75,80.0)$ & $74(57.25,82.75)$ & 0.065 \\
\hline APACHE-II score, M (IQR) & $12.5(8.0,18.0)$ & $12.0(8.0,18.0)$ & 0.421 \\
\hline SOFA score, M (IQR) & $4(2.75,7.0)$ & $4.5(2.0,8.0)$ & 0.731 \\
\hline Smoker, n (\%) & $56(43.08)$ & $52(46.43)$ & 0.601 \\
\hline Respiratory, n (\%) & 35 (26.92) & $42(37.5)$ & 0.105 \\
\hline Cardiology, n (\%) & 45 (34.62) & $40(35.71)$ & 0.858 \\
\hline Hypertension, n (\%) & $59(45.38)$ & $43(38.39)$ & 0.272 \\
\hline Hematological tumor, n (\%) & $16(12.31)$ & $6(5.36)$ & 0.100 \\
\hline Solid tumor, n (\%) & $20(15.38)$ & $24(21.43)$ & 0.224 \\
\hline
\end{tabular}

M-ROSE, microbiological rapid on-site evaluation; APACHE-II, Acute Physiology and Chronic Health Evaluation; SOFA, Sequential Organ

Failure Assessment; BMI, body mass index.

Table 2 Inflammatory indicators and treatment changes

\begin{tabular}{|c|c|c|c|}
\hline Variable & M-ROSE (n=130), M (IQR) (days) & Control (n=112), M (IQR) (days) & $P$ value \\
\hline IL-6 returned to normal & $8(2.0,12.5)$ & $8(4.5,15.5)$ & 0.067 \\
\hline CRP initial decrease & $1(1.0,1.0)$ & $1(1.0,5.0)$ & $<0.001$ \\
\hline CRP returned to normal & $8(4.0,10.75)$ & $9(5.0,15.0)$ & 0.064 \\
\hline PCT returned to normal & $1(1.0,3.0)$ & $3(1.0,8.0)$ & 0.006 \\
\hline Temperature initial decrease & $1(1.0,2.0)$ & $1(1.0,4.0)$ & 0.265 \\
\hline Temperature returned to normal & $2(1.0,5.0)$ & $1(1.0,5.0)$ & 0.712 \\
\hline Types of antibiotics used & $5(3.0,5.0)$ & $3(2.0,4.0)$ & $<0.001$ \\
\hline
\end{tabular}

M-ROSE, microbiological rapid on-site evaluation; CRP, C-reactive protein; PCT, procalcitonin. 
rate, ICU mortality, or hospitalization days between the 2 groups $(\mathrm{P}>0.05$, Table 3$)$.

\section{The effect of M-ROSE on the changes in inflammatory indicators and the prognosis of patients in different subgroups}

As shown in Table 4, for patients on invasive mechanical ventilation, the mortality rate $(48.44 \%)$ of the M-ROSE group was significantly lower $(\mathrm{P}<0.05)$ than that of the control group (69.44\%). For patients on not invasive mechanical ventilation, although there was no significant difference in mortality between the 2 groups, the time for the inflammatory index IL-6 and PCT to return to normal and the time for CRP and PCT to start decreasing in the $M-R O S E$ group were significantly shorter than those in the control group $(\mathrm{P}<0.05)$. Using the BALF culture results as the standard, the patients were divided into bacterial infection and fungal infection subgroups. As shown in

Table 3 Primary outcomes

\begin{tabular}{lccc}
\hline Variable & M-ROSE $(n=130)$ & Control $(n=112)$ & $P$ value \\
\hline Hospitalization days, & $19(13.0,30.0)$ & $16.5(10.25,32.0)$ & 0.219 \\
M (IQR) & & & \\
28-day mortality, $n(\%)$ & $28(21.54)$ & $19(16.96)$ & 0.370 \\
ICU mortality, $n(\%)$ & $42(32.31)$ & $35(31.25)$ & 0.744 \\
\hline
\end{tabular}

M-ROSE, microbiological rapid on-site evaluation; ICU, intensive care unit.
Table 5, for the patients with bacterial infection, the time for CRP and PCT to start decreasing and the time for PCT to return to normal were significantly shorter in the M-ROSE group $(\mathrm{P}<0.05)$. For patients with fungal infection, there was no significant difference in the changes in inflammatory indicators and prognosis between the 2 groups.

\section{Comparison between M-ROSE and laboratory results}

A total of 225 clinical specimens from 130 patients in the M-ROSE group were included in the study and sent to the laboratory for smear and bacterial and fungal culture. Of these, 71 specimens underwent mNGS. As shown in Figure 2A,2B, M-ROSE was consistent with the results of the smear in 96 specimens (42.7\%). Compared with smear (48.4\%), the correspondence rate between the results of M-ROSE and bacterial and fungal culture (57.3\%) was higher. Also, compared with smear (42.7\%) and culture (64.8\%), the correspondence rate between the results of M-ROSE and $\mathrm{mNGS}$ (66.2\%) was higher.

Furthermore, as shown in Figure $2 C-2 E$, the reporting time for M-ROSE results was faster than that for the smear results by an average of $3.56 \pm 6.09$ hours, faster than the bacterial and fungal culture results by $3.61 \pm 1.28$ days, and faster than the mNGS results by $1.64 \pm 1.07$ days. These differences were statistically significant $(\mathrm{P}<0.05)$.

\section{Discussion}

In the ICU, more than $51 \%$ of critically ill patients are

Table 4 Inflammatory indicator changes and prognosis between patients on invasive mechanical ventilation and not invasive mechanical ventilation in the 2 groups

\begin{tabular}{|c|c|c|c|c|c|c|}
\hline Variable & \multicolumn{3}{|c|}{ Patients on not invasive mechanical ventilation } & \multicolumn{3}{|c|}{ Patients on invasive mechanical ventilation } \\
\hline IL-6 initial decrease (days), M (IQR) & $1(1.0,2.0)$ & $1(1,4.25)$ & 0.07 & $1(1.0,2.0)$ & $1(1.0,10.0)$ & 0.406 \\
\hline IL-6 returned to normal (days), M (IQR) & $7(1.0,11.0)$ & $8(5,15.25)$ & 0.035 & $9(3.5,13.5)$ & $10(3,18)$ & 0.669 \\
\hline CRP initial decrease (days), M (IQR) & $1(1.0,1.0)$ & $1.5(1.0,5.0)$ & 0.001 & $1(1,1.5)$ & $1(1.0,6.0)$ & 0.178 \\
\hline PCT initial decrease (days), M (IQR) & $1(1.0,1.0)$ & $1(1.0,3.0)$ & $<0.001$ & $1(1.0,1.0)$ & $1(1.0,3.0)$ & 0.669 \\
\hline PCT returned to normal (days), M (IQR) & $1(1.0,1.0)$ & $3(1.0,8.0)$ & $<0.001$ & $3(1,8.5)$ & $2(1.0,4.0)$ & 0.936 \\
\hline 28-day mortality rate, $\mathrm{n}(\%)$ & $9(13.64)$ & $5(6.6)$ & 0.159 & $19(29.69)$ & $14(38.89)$ & 0.348 \\
\hline Mortality rate, $\mathrm{n}(\%)$ & $11(16.67)$ & $10(13.16)$ & 0.557 & $31(48.44)$ & 25 (69.44) & 0.042 \\
\hline
\end{tabular}

M-ROSE, microbiological rapid on-site evaluation; CRP, C-reactive protein; PCT, procalcitonin. 
Table 5 Inflammatory indicator changes and prognosis between patients with bacterial infection and fungal infection in the 2 groups

\begin{tabular}{|c|c|c|c|c|c|c|}
\hline Variable & \multicolumn{3}{|c|}{ Patients with bacterial infection } & \multicolumn{3}{|c|}{ Patients with fungal infection } \\
\hline IL-6 initial decrease (days), M (IQR) & $1(1.0,2.0)$ & $1(1.0,5.0)$ & 0.083 & $1(1.0,2.0)$ & $1.5(1,4.5)$ & 0.345 \\
\hline IL-6 returned to normal (days), M (IQR) & $8(2,12.5)$ & $8(5,15.5)$ & 0.098 & $3(1.0,10.0)$ & $10(2,17.25)$ & 0.193 \\
\hline CRP initial decrease (days), M (IQR) & $1(1.0,1.0)$ & $1(1.0,5.0)$ & $<0.001$ & $1(1.0,2.0)$ & $1.5(1.0,5.0)$ & 0.247 \\
\hline PCT initial decrease (days), M (IQR) & $1(1.0,1.0)$ & $1(1.0,3.0)$ & $<0.001$ & $1(1.0,1.0)$ & $1(1.0,1.0)$ & 0.808 \\
\hline PCT returned to normal (days), M (IQR) & $1(1.0,3.0)$ & $3(1.0,8.0)$ & 0.002 & $1(1,6.5)$ & $1(1.0,8.0)$ & 0.972 \\
\hline 28-day mortality rate, $\mathrm{n}(\%)$ & $25(21.74)$ & $17(17.35)$ & 0.422 & $3(20)$ & $2(14.29)$ & 0.684 \\
\hline Mortality rate, $\mathrm{n}(\%)$ & $36(31.3)$ & $33(33.67)$ & 0.713 & $6(40)$ & $2(14.29)$ & 0.122 \\
\hline
\end{tabular}

M-ROSE, microbiological rapid on-site evaluation; CRP, C-reactive protein; PCT, procalcitonin.

A

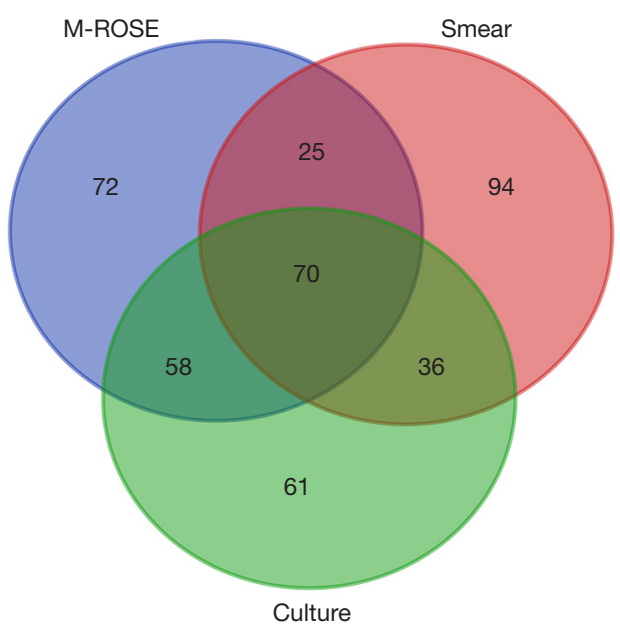

C

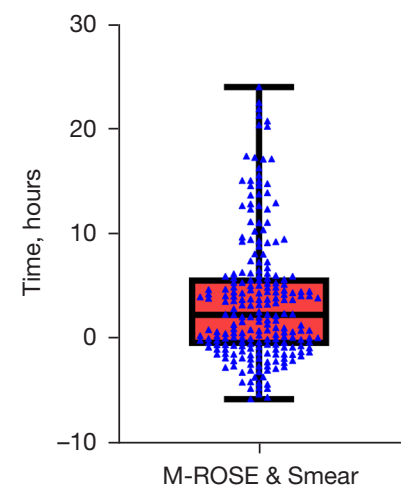

B
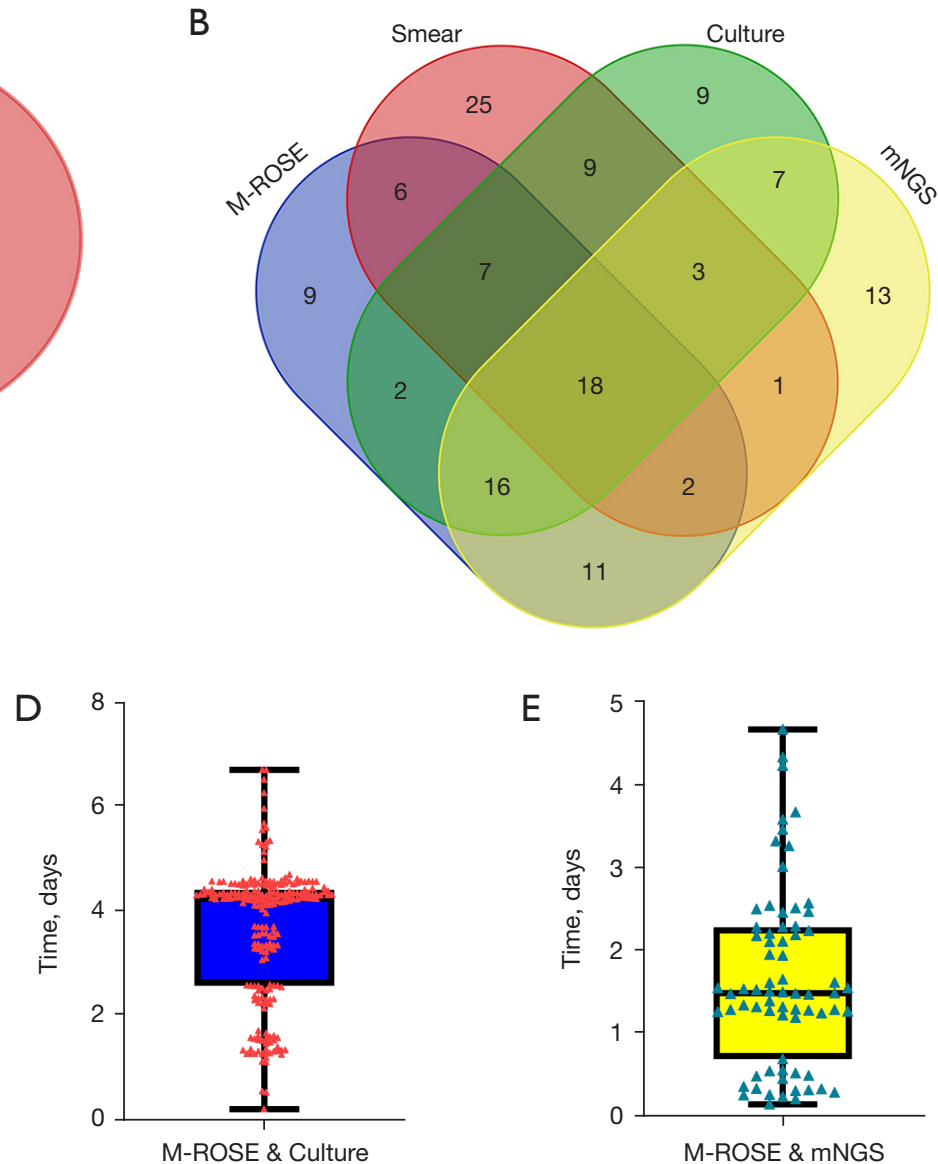

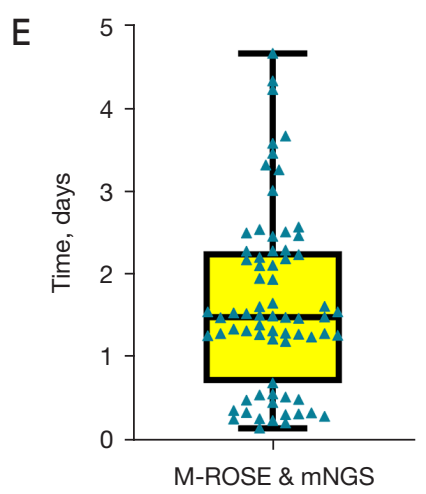

Figure 2 Venn diagrams for M-ROSE, smear, culture, and mNGS results $(A, B)$ and box plots of the time difference between the availability of M-ROSE and laboratory test results (C-E). M-ROSE, microbiological rapid on-site evaluation; mNGS, metagenomic next-generation sequencing. 
diagnosed with an infection, $64 \%$ of which are respiratory tract infections. Once diagnosed with an infection, the ICU mortality rate has been shown to double (10-12). In the RICU, the proportion of lower respiratory tract infections is high, and patients' conditions change rapidly. The most important factor is the rapid diagnosis of pathogens in the early stage. At present, the most commonly used microbial identification methods in clinical practice include bacterial and fungal culture, antigen-antibody detection, mass spectrometry, and nucleic acid amplification PCR, among others. However, these methods are generally time consuming and expensive, which may lead to a lag in pathogen diagnosis and delayed treatment. The positive rate of pulmonary fungal infection culture results is low, and the traditional culture method generally requires 3 to 5 days or even longer and cannot provide the evidence needed for an early clinical diagnosis.

ROSE is a rapid, real-time cytological interpretation technique that accompanies the sampling process. In Europe and the United States, C-ROSE is widely used in diagnostic interventional pulmonology due to its high diagnosis rate for malignant tumors, its ability to diagnose infectious and noninfectious diseases, and its low complications (13-16). However, there are few studies on the diagnostic value of M-ROSE as a microbiological evaluation technique for determining the pathogenesis of pulmonary infections. This study retrospectively included 130 patients in the M-ROSE group and 112 patients in the control group, and the data of the 2 groups were comparable. The inflammatory indicators in the M-ROSE group, including the time until IL-6, CRP, and PCT started to decline and the time until these inflammatory indicators returned to normal, were significantly lower than those in the control group, indicating that M-ROSE-assisted diagnosis in the RICU was conducive to alleviating inflammation and slowing disease progression. The M-ROSE group used significantly more types of antibiotics than the control group. This may be due to the current routine empirical use of broad-spectrum antibiotics in clinical practice and the fact that the identification of new pathogenic microbial species by M-ROSE has resulted in an increase in the types of antibiotics administered. M-ROSE has the ability to identify pathogens that are missed by clinical empirical judgment, thus increasing the use of appropriate and effective antimicrobial drugs.

For patients on invasive mechanical ventilation, M-ROSE can help improve prognosis and reduce the mortality rate. For patients on not invasive mechanical ventilation and patients with bacterial infections, M-ROSE helps to promote a decrease in inflammatory indicators. Patients on invasive mechanical ventilation are usually more critically ill and have more complicated pulmonary conditions than other patients. Especially in the RICU, pulmonary infection is often associated with the colonization of multidrug-resistant bacteria. Therefore, it is particularly important to distinguish between bacterial infection and colonization and to determine the infective pathogen. Bedside M-ROSE helps clinicians quickly and accurately determine the cytological pattern of the lower respiratory tract, distinguish between bacterial colonization and infection, and understand the progression of lung diseases. However, patients with fungal infection usually have a long treatment cycle and no significant increase in inflammatory indicators. Therefore, although M-ROSE can be used to diagnose fungal infection at an early stage, the inflammatory indicator levels may not be significantly decreased, and the prognosis of these patients may not be significantly improved during hospitalization. Further expansion of the sample size is needed to clarify the effect of M-ROSE on the prognosis of patients with fungal infection.

A comparison between the results of M-ROSE and laboratory testing highlighted the high accuracy of M-ROSE. Additionally, the available time for the M-ROSE results was significantly shorter than for clinical smear, culture, and mNGS results. These results confirm the value of M-ROSE for the early and rapid diagnosis of lower respiratory tract infections, which is conducive to early (within 1 hour) guidance regarding antibiotic regimens for critically ill patients and, consequently, the coverage of targeted pathogens, treatment of pulmonary infection, and improved outcomes.

M-ROSE is a microbial diagnostic technique with a low cost, simple operation, and important clinical value. Only a microscope, 2 staining reagents, and a small number of specimens are needed to perform bedside M-ROSE. Operators can complete specimen preparation, smearing, and staining after a short training class. Basic cytology and microbiology interpretation can be performed after systematic training. The artificial intelligence identification platform for lower respiratory tract pathogenic microorganisms, which is being developed by our department, can automatically identify cells, bacteria, and fungi. It can also determine whether BALF specimens are appropriate and whether there are bacterial and fungal infections, classify infected bacteria, and determine the 
amount of bacteria within 5 minutes. Additionally, it can solve the problem of inconsistent reliability of M-ROSE results due to lack of experience. We hope that it can be made available to primary hospitals to help medical staff perform automatic bedside M-ROSE analysis in the future. Although the diagnostic value of BALF is not as good as that of lung tissue specimens, BALF specimens are easier to obtain, and distal airway and alveolar epithelial secretions can be collected to determine the nature of the lesion and possible pathogens. The BALF should be sampled in strict accordance with guidelines. The purpose of M-ROSE is to identify the actual infectious pathogens. The observation of obvious phagocytosis of neutrophils or macrophages under microscopy has clear implications for the diagnosis of pathogenic microorganisms in lower respiratory tract infections. The diagnosis of hyphae is relatively easy with M-ROSE, which has high sensitivity and specificity $(17,18)$.

However, M-ROSE analysis has certain limitations. It is difficult to identify the species of microorganism based on the morphology and many pathogens such as virus and mycoplasma can not be observed. Besides, M-ROSE may aid to improve the current status of ICU crowded with drug resistant bacteria while it can not directly reflect drug sensitivity for drug resistant bacteria. Combination of M-ROSE and metagenomics next-generation sequencing may be a way to detect more pathogens and offer the drug resistance gene phenotype to guide more accurate antibiotics therapies $(19,20)$.

\section{Conclusions}

In summary, our study firstly demonstrate how the M-ROSE is beneficial for the diagnosis and prognosis of patients in RICU. The use of M-ROSE in RICU can aid in the early and rapid diagnosis of pathogenic microorganisms, guide antibiotic regimens for critically ill patients, ameliorate disease progression, and reduce mortality in patients on invasive mechanical ventilation. And we will further investigate the impact of M-ROSE on the mortality rate, length of hospitalization, and antibiotic use by rigorously designed prospective randomized controlled clinical trials.

\section{Acknowledgments}

Funding: The study was supported by Key Projects of Military Logistics Scientific Research Program (BLB18J008).

\section{Footnote}

Reporting Checklist: The authors have completed the STROBE reporting checklist. Available at https://dx.doi. org/10.21037/atm-21-5465

Data Sharing Statement: Available at https://dx.doi. org/10.21037/atm-21-5465

Conflicts of Interest: All authors have completed the ICMJE uniform disclosure form (available at https://dx.doi. org/10.21037/atm-21-5465). All authors report that the study was supported by Key Projects of Military Logistics Scientific Research Program (BLB18J008). The authors have no other conflicts of interest to declare.

Ethical Statement: The authors are accountable for all aspects of the work in ensuring that questions related to the accuracy or integrity of any part of the work are appropriately investigated and resolved. All procedures performed in this study involving human participants were in accordance with the Declaration of Helsinki (as revised in 2013). This study was approved by the Ethics Committee of Chinese PLA General Hospital (No. 2019-083-01). Individual consent for this retrospective analysis was waived.

Open Access Statement: This is an Open Access article distributed in accordance with the Creative Commons Attribution-NonCommercial-NoDerivs 4.0 International License (CC BY-NC-ND 4.0), which permits the noncommercial replication and distribution of the article with the strict proviso that no changes or edits are made and the original work is properly cited (including links to both the formal publication through the relevant DOI and the license). See: https://creativecommons.org/licenses/by-nc-nd/4.0/.

\section{References}

1. Meyer KC, Raghu G, Baughman RP, et al. An official American Thoracic Society clinical practice guideline: the clinical utility of bronchoalveolar lavage cellular analysis in interstitial lung disease. Am J Respir Crit Care Med 2012;185:1004-14.

2. Gasparini S. It is time for this 'ROSE' to flower. Respiration 2005;72:129-31.

3. Chow FW, Chan TT, Tam AR, et al. A Rapid, Simple, Inexpensive, and Mobile Colorimetric Assay COVID-19LAMP for Mass On-Site Screening of COVID-19. Int J 
Mol Sci 2020;21:5380.

4. Daigle J, Onyilagha C, Truong T, et al. Rapid and highly sensitive portable detection of African swine fever virus. Transbound Emerg Dis 2021;68:952-9.

5. Huang C, Wen T, Shi FJ, et al. Rapid Detection of IgM Antibodies against the SARS-CoV-2 Virus via Colloidal Gold Nanoparticle-Based Lateral-Flow Assay. ACS Omega 2020;5:12550-6.

6. Davenport RD. Rapid on-site evaluation of transbronchial aspirates. Chest 1990;98:59-61.

7. Speers DJ. Clinical applications of molecular biology for infectious diseases. Clin Biochem Rev 2006;27:39-51.

8. Oki M, Saka H, Kitagawa C, et al. Rapid on-site cytologic evaluation during endobronchial ultrasound-guided transbronchial needle aspiration for diagnosing lung cancer: a randomized study. Respiration 2013;85:486-92.

9. Beveridge TJ. Use of the gram stain in microbiology. Biotech Histochem 2001;76:111-8.

10. Vincent JL, Rello J, Marshall J, et al. International study of the prevalence and outcomes of infection in intensive care units. JAMA 2009;302:2323-9.

11. Sakr Y, Moreira CL, Rhodes A, et al. The impact of hospital and ICU organizational factors on outcome in critically ill patients: results from the Extended Prevalence of Infection in Intensive Care study. Crit Care Med 2015;43:519-26.

12. Vincent JL, Marshall JC, Namendys-Silva SA, et al. Assessment of the worldwide burden of critical illness: the intensive care over nations (ICON) audit. Lancet Respir Med 2014;2:380-6.

Cite this article as: Tao Y, Song L, Fu H, Zhang W, Song Y, Liu H, Xie L, Wang K. Application of microbiological rapid onsite evaluation in respiratory intensive care units: a retrospective study. Ann Transl Med 2022;10(1):7. doi: 10.21037/atm-215465
13. Trisolini R, Cancellieri A, Tinelli C, et al. Rapid on-site evaluation of transbronchial aspirates in the diagnosis of hilar and mediastinal adenopathy: a randomized trial. Chest 2011;139:395-401.

14. Griffin AC, Schwartz LE, Baloch ZW. Utility of onsite evaluation of endobronchial ultrasound-guided transbronchial needle aspiration specimens. Cytojournal 2011;8:20.

15. Kalanjeri S, Gildea TR. Electromagnetic Navigational Bronchoscopy for Peripheral Pulmonary Nodules. Thorac Surg Clin 2016;26:203-13.

16. Umeda Y, Otsuka M, Nishikiori H, et al. Feasibility of rapid on-site cytological evaluation of lung cancer by a trained pulmonologist during bronchoscopy examination. Cytopathology 2019;30:628-33.

17. Sosa Fernandez LV, Plaitano F, Varone V, et al. Lymph node fine needle cytology in the diagnosis of infectious diseases and ancillary techniques. Infez Med 2012;20 Suppl 3:21-5.

18. Baselski VS, Wunderink RG. Bronchoscopic diagnosis of pneumonia. Clin Microbiol Rev 1994;7:533-58.

19. Rose G, Wooldridge DJ, Anscombe C, et al. Challenges of the Unknown: Clinical Application of Microbial Metagenomics. Int J Genomics 2015;2015:292950.

20. Hernando-Amado S, Coque TM, Baquero F, et al. Defining and combating antibiotic resistance from One Health and Global Health perspectives. Nat Microbiol 2019;4:1432-42.

(English Language Editor: C. Betlazar-Maseh) 